High-dose olanzapine in treatment resistant schizophrenia. A case report and literature review

Ciara Clarke*, Clodagh Rushe and Fintan Byrne

Mayo University Hospital

${ }^{\star}$ Corresponding author.

doi: 10.1192/bjo.2021.335

Objective. We report a case of a 58-year-old gentleman who was hospitalised intermittently for one year due to treatment resistant schizophrenia. Prior to hospitalisation he had been prescribed standard antipsychotics for decades without full resolution of positive psychotic symptoms. During his final admission lasting six months he was guarded, suspicious, irritable, constantly paced the corridor and displayed thought block and paranoid persecutory delusions. He would not enter the assessment room or allow any blood or ECG monitoring, however, he was compliant with oral medication. He was successfully treated with high dose olanzapine $(40 \mathrm{mg} /$ day) and was discharged to the community. The aim of this study is to bring awareness and add to the body of evidence for the use of high-dose olanzapine in patients with treatment resistant schizophrenia in whom a trial of clozapine is not possible.

Case report. The patient gave written consent for this case report to be written and presented. An extensive literature review was performed and key papers were identified. Discussion focuses on the key areas in the literature.

Discussion. This case demonstrates that high-dose olanzapine can be used effectively as an alternative to clozapine in treatment resistant schizophrenia.

Conclusion. This case highlights the need for further evaluation of high-dose olanzapine as an alternative to clozapine in patients with treatment-resistant schizophrenia.

\section{Facilitated early discharge in Wandsworth}

Mudasir Firdosi*, Allerdiena Hubbeling and Twaisha Kapoor

St Georges University of London

${ }^{*}$ Corresponding author.

doi: 10.1192/bjo.2021.336

Objective. There is limited research surrounding facilitated early discharge (FD) and Home Treatment Teams (HTTs). This study aimed to compare patients who received FD with patients who were discharged without FD to identify whether there were significant differences in terms of social demographics, illness characteristics, health outcome and treatment duration. Using this data we furthermore aimed to provide proposals to help advance the effectiveness of $\mathrm{FD}$, as well as suggesting concepts of where future research should lie.

Case report. A randomised sample of patients who received FD and patients who were discharged without FD was obtained from a South London Hospital. This was manually narrowed down to patients specifically treated by the Wandsworth Home Treatment Team (WHTT). Socio-demographic and clinical data were then attained from the patients' electronic records to compare and statistically analyse between the two groups.

Discussion. Patients who received FD from the WHTT were found to have significantly less previous psychiatric admissions compared to those who were discharged without FD $(p=0.032)$. All other variables were found to have no association with FD.

Conclusion. Having a high number of previous psychiatric admissions seems to be an aspect that decreases the chance of being allocated FD. This variable can be seen as an indicator of severity of illness and a challenging social environment; it could therefore be valuable to take this variable into consideration when allocating FD. Furthermore, total treatment duration was found to not be significantly different for FD and non-FD patients, thus supporting the use of CRHTTs as an equivalent alternative for inpatient admission, however, national scale research should be conducted to strengthen and expand on these findings.

\section{Obsessive compulsive disorder: a case of extreme obsessional slowness in an 18-year-old presenting to the national OCD unit}

Claire Fischer*, Ilenia Pampaloni and Sarah Gardiner

South West London and St George's Mental Health Trust ${ }^{\star}$ Corresponding author.

doi: $10.1192 /$ bjo.2021.337

Objective. Obsessional slowness in OCD is a rare phenomenon on which there is minimal published literature. This is a particularly severe and atypical case of early onset OCD with extreme obsessional slowness and mutism. To the best of our knowledge, there have been no reports of similar severity published in this age group. This report seeks to provide discussion of important organic causes that may need to be considered as well as information on treatment approach.

Case report. An 18-year-old male was admitted to the National OCD Unit, Springfield Hospital with a history of autism and normal development until the age of 14 , after which symptoms of OCD with fear of contamination emerged, followed by progressive motor slowness and mutism.

Due to the severity of OCD and self-neglect he had two previous admissions to CAMHS wards and required a course of ECT to treat catatonic symptoms age 17.

Pharmacological treatment has included Aripiprazole $5 \mathrm{mg}$ and Fluoxetine $60 \mathrm{mg}$, which the patient was taking at admission. The latter was subsequently switched to Sertraline $250 \mathrm{mg}$ and Aripiprazole increased. As it was hypothesized that his obsessional slowness stemmed from severe levels of anxiety, Buspirone was also added.

Therapy has been intensive, although communication difficulties have made targeting specific fears challenging as the exact nature of the intrusive thoughts remains unclear.

Discussion. Following combined neurology and neuropsychiatry review, the patient spent four weeks in a general hospital for further investigation as it was initially felt an organic cause was likely. Initial differentials included Juvenile Onset Parkinson's or Wilson's disease. Both were subsequently ruled out and despite multiple investigations, no obvious organic cause was found. A markedly abnormal FDG PET scan showed findings usually seen in advanced dementia, but not necessarily clinically correlating to his current presentation.

The OCD unit have continued to provide intensive input and tailored treatment programme, encouraging actions against any rules he has in place. Prompting and pacing, verbal exercises and regular stretching exercises due to stooped posture which he attributed to needing to obey certain rules have been used.

Conclusion. It is important for clinicians to be aware of obsessional slowness in OCD and this report highlights a particularly rare and severe example in a young adult who has been difficult to treat. Organic causes may need to be considered and MDT approach to treatment is essential. 
An unregistered TARDBP mutation in a case presenting with young-onset dementia

\author{
Mahmoud Gad ${ }^{1 \star}$, Walid Nasr ${ }^{1}$ and Tareq Qassem ${ }^{2}$ \\ ${ }^{1}$ Al-Amal Hospital, Ministry of Health and Prevention and \\ ${ }^{2}$ Maudsley Health, Al-Amal Hospital, Ministry of Health and \\ Prevention, Mohammed Bin Rashid University Of Medicine and \\ Health Sciences, Institute of Psychiatry, Ain Shams University \\ ${ }^{*}$ Corresponding author.
}

doi: 10.1192/bjo.2021.338

Objective. This poster aims to report an unregistered mutation in Transactive Response DNA Binding Protein (TARDBP) gene in a patient presenting young-onset dementia.

Hypothesis: Novel heterozygous mutation in the TARDBP gene is linked to a case of with young-onset dementia.

Background. Pathogenic variants in TARDBP cause autosomal dominant fronto-temporal degeneration, characterized by TDP43-positive inclusions, dystonia, dyslexia, receptive dysphasia, and paraphrasic errors. In addition to the neurocognitive deficits, patients might suffer from cardiomyopathy and amyotrophic lateral sclerosis.

Case report. Molecular genetic analysis of whole-exome sequencing (WES) was carried out for a 45 -year-old male patient presenting with cognitive decline and behavioural symptoms.

Discussion. WES Identified the heterozygous variant c.527A $>\mathrm{T}$ p.(Lys 176lle) in TARDBP gene. To the best of our knowledge the variant has not been described in the literature so far (HGMD 2019.3). No allele frequencies in the general population have been documented.

Conclusion. We believe that we have identified a novel mutation in the TARDBP gene. This mutation is likely to be linked to this patient presenting with young-onset dementia.

\section{Off-licence use of clozapine in patients with emotionally unstable personality disorder: a case series analysis

\author{
Declan Hyland ${ }^{1 *}$, Alex Walmsley ${ }^{2}$ and Victoria Simpson ${ }^{2}$ \\ ${ }^{1}$ Consultant Psychiatrist, Clock View Hospital, Liverpool, Mersey \\ Care NHS Foundation Trust and ${ }^{2} 5$ th year medical undergraduate, \\ University of Liverpool \\ ${ }^{\star}$ Corresponding author.
}

\section{doi: 10.1192/bjo.2021.339}

Objective. This retrospective case series followed emotionally unstable personality disorder (EUPD) patients following initiation of clozapine on an off-licence basis, aiming to examine tolerance by determining side effect prevalence and treatment cessation frequency, as well as examining efficacy, by investigating number of hospital re-admissions and symptom control.

Case report. This case series captured the experiences of 11 EUPD patients under the care of Mersey Care NHS Foundation Trust, all of whom had, at some time in the past five years, been initiated on clozapine. All patients were white British females, with a median age of 31 . The median daily dose of clozapine was $300 \mathrm{mg}$. Most patients had significant psychiatric comorbidities, as well as illicit substance and / or alcohol misuse.

Whilst prescribed clozapine, patients were only admitted to hospital once on average and this was commonly for clozapine re-titration. Whilst in hospital, rates of self-harm were low, but ligaturing and suicide attempts showed higher prevalence. Patients still demonstrated self-harming behaviour out of hospital leading to $\mathrm{A}$ and $\mathrm{E}$ presentations. In the community, contacts with the police were minimal, with only two patients undergoing Section 136 assessments or arrests.

All patients reported side effects from clozapine - usually hypersalivation, over-sedation and constipation. All 11 patients experienced sinus tachycardia. Eight patients temporarily ceased taking clozapine at some point. In three patients, discontinuation of clozapine was as a result of intolerable side effects. Three patients experienced neutropenia, which subsequently resolved. Only two patients had a body mass index within healthy range. Discussion. Despite patients reporting clozapine to provide symptomatic benefit for their EUPD, and improved their engagement with mental health services, prevalence of self-harm and of $\mathrm{A}$ and $\mathrm{E}$ presentations remained high, indicating the importance of community support and concomitant psychotherapeutic treatment. Patients with more robust community support showed greater adherence to clozapine.

High prevalence of side effects and obesity in these patients, in addition to risk of developing neutropenia, highlights the importance of rigorous monitoring after initiating clozapine. It is reassuring that, despite development of neutropenia in some patients, this recovered quickly, and clozapine treatment could resume.

Conclusion. Clozapine may be an effective pharmacological treatment for enabling EUPD patients to engage more therapeutically with services. Clozapine may be of greater benefit to those with more stable, less chaotic lives. Although diminished, patients still show self-harming behaviour and need for A and E admissions and re-hospitalisation. Side effects of clozapine are common and regular monitoring is required.

\section{A haven for an extremely disturbed young person}

\section{Rummana Khan*}

Consultant Child \& Adolescent Psychiatrist, Leicester Partnership NHS Trust

${ }^{*}$ Corresponding author.

doi: 10.1192/bjo.2021.340

Objective. The reason to share this case is to high light the lack of resources in mental health services which can delay the provision of appropriate care and this can have negative impact on child health outcomes.

Background. A 10-year-old boy was referred to CAMHS. He presented with extremely challenging behaviours. After first appointment with CAMHS he attacked his father and nurses. He had to be restrained multiple times. He started to use wooden chair as a weapon, threatened to harm others and threatened to urinate on staff. He tried to kill him-self by ligature. Mental health act assessment was completed and when a decision was reached that detention under the mental health act was appropriate, no appropriate bed was available. He was admitted under Section II of MHA to paediatric ward where he remained for one week (with 2:1 CAMHS support). Then he was transferred to an inpatient CAMHS unit which was commissioned for children over 12 years of age. At a later date mental health tribunal panel upheld the section. After few days he was transferred to an age appropriate in-patient mental health bed. He stayed there for roughly 6 months and was discharged with a diagnosis of ADHD and Autistic Spectrum Disorder. There was a long delay in discharge, until appropriate specialist residential placement could be identified and he was transferred there. He is well settled now in the placement.

Case report. Legal advice was later taken on this case. MHA 1983, Human Rights Act, Children Act 1989, Criminal Law Act 1967 and Code of Practice 2015 were considered and it was agreed that it was appropriate to use MHA 1983. There was discussion whether the Children Act could be relied on instead, but in 